\title{
Aggregation of Hydroacoustic Monitoring System
}

\author{
Pavel A. Starodubtsev*, \\ Evgeny A. Storozhok and Roman N. Alifanov \\ Pacific Higher Naval School named after S.O. Makarov \\ Vladivostok, Russian Federation
}

Received 03.11.2019, received in revised form 11.12.2019, accepted 19.02.2020

\begin{abstract}
Currently, the threat of sabotage at hydraulic structures such as bridges, dams, oil rigs, etc. has increased. In this regard, the creation of effective hydroacoustic monitoring systems is an urgent task. Aggregation (from Latin: agrego) means a combination of individual elements into a single entity. In relation to the problem considered, this term refers to the combination of states of an object or, more precisely, a group of objects that possess common properties. The article presents an aggregation method using a single criterion which characterizes the minimum of information loss.
\end{abstract}

Keywords: hydroacoustic monitoring system, degree of goal achievement, tuple, system aggregation operator, membership function, information losses.

\section{Агрегирование системы}

\section{гидроакустического мониторинга}

\author{
П.А. Стародубцев, Е.А. Сторожок, Р.Н. Алифанов* \\ Тихоокеанское высшее военно-морское училище им. С.О. Макарова \\ Российская Федерация, Владивосток
}

Аннотащия. В настоящее время возросла угроза диверсий на гидротехнических сооружениях (мосты, плотины, буровые вышки и т.п.). В связи с этим создание эффективных систем гидроакустического мониторинга является актуальной задачей. Агрегирование (от лат. agrego) - объединение отдельных элементов в одно целое. Применительно к рассматриваемой проблеме этим термином обозначается объединение ряда состояний объекта или, точнее, группы объектов, обладающих теми или иными общими свойствами. В статье представлен

(C) Siberian Federal University. All rights reserved

This work is licensed under a Creative Commons Attribution-NonCommercial 4.0 International License (CC BY-NC 4.0).

* Corresponding author E-mail address: spa1958@mail.ru 
метод агрегирования с использованием единого критерия, характеризующего минимум потерь информации.

Ключевые слова: система гидроакустического мониторинга, степень достижения цели, кортеж, оператор агрегирования системы, функция принадлежности, информационные потери.

Цитирование: Стародубцев, П.А. Агрегирование системы гидроакустического мониторинга / П.А. Стародубцев, Е.А. Сторожок, Р.Н. Алифанов // Журн. Сиб. федер. ун-та. Техника и технологии, 2020. 13(2). С. 206-212. DOI: 10.17516/1999-494X-0217

Проблема агрегирования системы гидроакустического мониторинга является сложной слабоструктурированной проблемой, решение которой требует учета множества факторов в условиях не поддающейся структуризации неопределенности. Причем неопределенность существует как в отношении среды, в которой система гидроакустического мониторинга должна функционировать, так и в отношении параметров системы и характеристик компонентов. По сути своей она относится к классу задач принятия решений в нечеткой обстановке. Разрешение неопределенности обуславливается возникновением проблемной ситуации, в которой имеются два состояния - существующее и желаемое, причем существует несколько способов достижения желаемого состояния. Принципиальная трудность выбора в подобных условиях заключается в неопределенности понятия «наилучший альтернативный вариант» $[1,2]$.

В основе формирования представления и выбора «наилучшего альтернативного варианта» принятия решения лежит процесс формулирования обоснования, структуризации и формализации цели. Цель при агрегировании сложных систем вначале возникает в виде некоторого «образа» или «области». В наибольшей степени это проявляется на уровне глобальной цели. При этом достичь единого понимания области цели принципиально невозможно без ее детализации в виде неупорядоченного или упорядоченного (в структуре) набора одновременно возникающих взаимосвязанных подцелей, которые делают ее более конкретной и понятной. Сказанное позволяет сделать вывод о том, что задача формирования обобщающей цели в сложных системах не только может, но и должна сводиться к задаче ее структуризации и формализации [3]. В основу представленного ниже материала положена гипотеза о возможности агрегирования этих компонентов с использованием единого показателя и критерия, характеризующего степень достижения цели.

Для структуризации и формализации подобного интегрального показателя уместно привести известный обобщенный подход формализации цели $[3,4]$. Формализованные цели могут быть четкими и размытыми. Если они имеют четкую область и четкую границу, то это означает, что вся область цели равнозначна и соответствует оптимальному функционированию, а переход за ее границу вызывает качественный скачок - цель не достигается. В системе гидроакустического мониторинга, относящейся к классу больших и сложных систем, цель функционирования имеет нечеткий, размытый характер, и эту размытость можно объяснить тем, что помимо области полного достижения и полного недостижения цели существует еще область ее частичного достижения. Кроме того, в отдельных случаях внутри области полного достижения цели могут существовать подобласти «перевыполнения» цели.

В общем случае (рис. 1) цель может задаваться в некотором $n$-мерном пространстве существенных параметров $x_{1}, x_{2}, \ldots, x_{n}[3,4]$. Идеальная точечная цель в этом пространстве опреде-

$$
-207-
$$



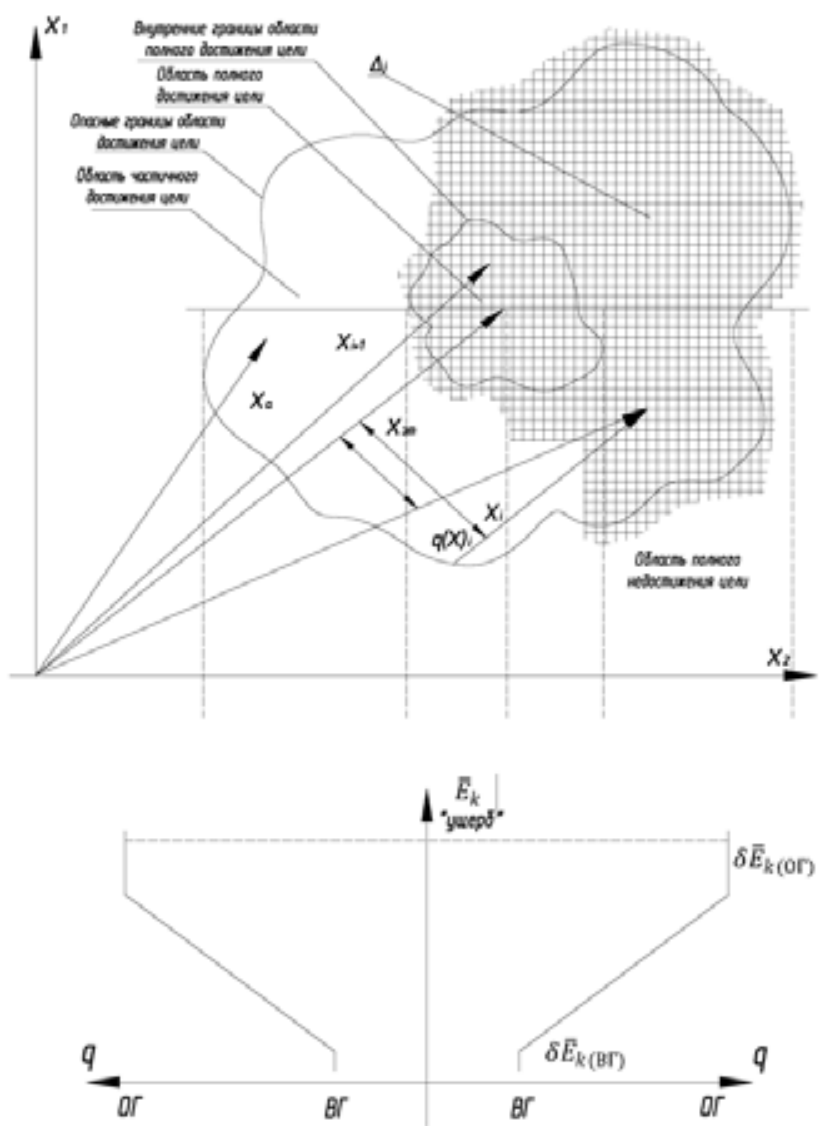

Рис. 1. Формализация цели

Fig. 1. Formalization of the target

ляется концом вектора цели $x_{n}$. Будем считать, что вся область цели характеризуется внешними «опасными» границами (ОГ) $\xi\left(x_{O \Gamma}\right)$ и «внутренними» границами (ВГ) $\xi\left(x_{B \Gamma}\right)$. За пределами ОГ цель полностью не достигается. В пределах ВГ с допустимой точностью цель полностью достигается. Между внешними «опасными» и «внутренними» границами существует область частичного достижения цели.

Наличие области частичного достижения цели является следствием широкого спектра факторов неопределенности и риска.

Опасные границы области цели имеют размытый характер, и поэтому их можно считать условно опасными. Выход за их пределы означает нарушение штатного функционирования системы, убыточность ее работы, отставание от программы функционирования, но все это еще не катастрофа.

В пространстве выделенных существенных параметров всю область цели обозначим $V_{\text {Оц, }}$ а область полного достижения цели - $V_{\text {Пц. }}$

При разбиении этих областей на дискреты $\Delta_{j}$ и их подсчете получим соответственно числа $N_{\text {Оц и }} N_{\text {пц}}$, которые характеризуют мощность множества всей области цели $M_{\text {Оц и мощность }}$ множества полного достижения цели $M_{\text {пц. }}$ 


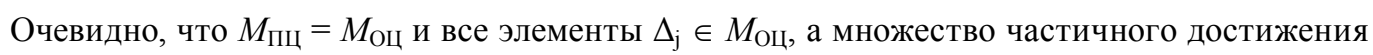

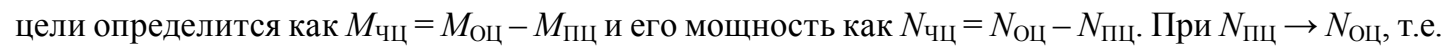
при $N_{\text {чц }} \rightarrow 0$, «опасные и «внутренние» границы области цели сливаются, и цель становится четкой.

Разбив область цели на элементы, будем считать, что если вектор $\dot{x}$ находится в каком-

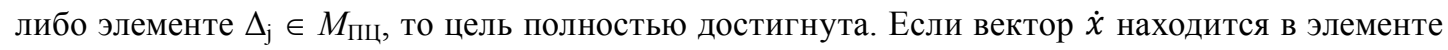
$\Delta_{\mathrm{j}} \in M_{\text {чц }}$, то будет отмечаться некоторое недостижение цели.

В общем случае степень достижения цели $\bar{Y}_{\text {кі }}$ определяется как функция удаленности і-го элемента $\left(\mathrm{q}_{\mathrm{i}}\right)$, в котором находится вектор $\dot{x}_{\mathrm{i}}$, от внутренней границы области цели

$$
\bar{Y}_{\mathrm{ki}}=\varphi\left[\mathrm{q}_{\mathrm{i}}-\xi\left(x_{\mathrm{B \Gamma}}\right)\right],
$$

где $\varphi$ - функция связи между $\bar{Y}_{\text {кі }}$ и удаленностью і-го элемента от ВГ в пространстве параметров области цели.

По характеру соотнесения координат конца вектора $\dot{x}_{\mathrm{i}} \mathrm{c} \xi\left(x_{\mathrm{B \Gamma}}\right)$ можно определить абсолютную, относительную и степенную виды степени достижения цели [3].

При векторном представлении степень достижения цели в n-мерном пространстве характеризуется модулем $\bar{Y}$ и совокупностью полярных углов $\left\{\alpha_{\mathrm{i}}\right\}, \mathrm{i}=1,2, \ldots, \mathrm{n}$, где $\mathrm{n}-$ размерность пространства цели.

Для практических расчетов удобно представить степень достижения цели в диапазоне $[0,1]$ как меру различия параметров в отношении их эталонов:

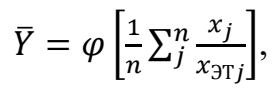

где $x_{\text {эт }}$ - эталонное значение ј-го параметра цели; $\mathrm{n}$ - размерность пространства цели, $\varphi$ - функция связи между $\bar{Y}_{\mathrm{ki}}$ и значениями параметров области цели $[3,4]$.

Таким образом, с учетом ориентации на достижение минимума потерь при агрегировании, что в свою очередь позволяет использовать его значение в качестве обратной связи при формировании и реконфигурации системы, а также с учетом приведенного подхода формализации целей при агрегировании предлагается представлять интегральный показатель степенью недостижения целей в диапазоне [0,1].

В условиях неопределенности процесс принятия решения при агрегировании системы описывается кортежем

$$
\left\langle F, S^{0}, R^{0}, l\right\rangle,
$$

где F - множество показателей, характеризующих систему; $\mathrm{S}^{\circ}$ - множество исходных компонентов для ее построения; $\mathrm{R}^{0}$ - множество действий разработчика или оператора агрегирования системы, $\mathrm{R}^{\circ}=\left(\mathrm{A}^{\circ}, \mathrm{S}\right), \mathrm{a}^{\circ} \in \mathrm{A}^{0}$ - действие, множество действий оператора по выбору компонентов; $l$ - функция, характеризующая потери при формировании облика системы, когда на отношении $\mathrm{F} \times \mathrm{R}^{\circ}$ действием $\mathrm{a}^{\circ} \in \mathrm{A}^{0}$ выбирается $f_{a} \in F[3,5]$.

Нечеткое действие $\mathrm{a}^{\circ} \in \mathrm{A}^{0}$ имеет функцию принадлежности $\mu_{\mathrm{A}}: A^{0} \rightarrow[0,1]$, а нечеткая стратегия построения $R_{a}^{0}$ описывается нечетким отношением, характеризующим варианты выбора оператором построения исходных компонентов $S^{0} \times A^{0}$, и имеет функцию принадлежности

$$
-209-
$$




$$
\mu_{\mathrm{Ra}}: S^{0} \times A^{0} \rightarrow[0,1] .
$$

Цель задачи выбора заключается в поиске стратегии построения, которая минимизирует нечеткую функцию потерь

$$
\mu \mathrm{Ra}: F \times R^{0} \rightarrow \min l .
$$

Таким образом, в качестве целевой функции при решении задачи агрегирования системы гидроакустического мониторинга (выбора варианта построения) как информационной системы логично выбрать функцию, характеризующую информационные потери, а в качестве критерия решения - ее минимум.

Отработанная практика построения систем гидроакустического мониторинга базируется на использовании методов интерактивного моделирования для формирования наиболее предпочтительного варианта размещения на местности элементов системы.

Для обеспечения сравнимости результатов оценки плодотворным подходом является подход, опирающийся на гипотезу о возможности формирования интегрального показателя, позволяющего осуществить целенаправленное ее построение как при математическом моделировании, так и при построении в местах применения по предназначению и при ее функционировании.

Пусть $S$ - перспективная интегрированная система гидроакустического мониторинга и абстрактная ее модель описывается соотношением

$$
S=\{R \times P \times O\} .
$$

Тогда цель функционирования G можно описать соотношением

$$
G:\{M \times U\} \times\{R \times P \times O\}=X_{r} .
$$

Представим ее отображение на временном континууме Т следующим соотношением:

$$
F_{K}: X \times\{R \times P \times O\} \times T \times K,
$$

где $K \in F$ - показатель эффективности; $R=R^{g} \times\left(R^{s} \times S\right)$ - оператор системы, $R^{s}=\left(A^{F}, R^{F}\right)$ - множество функционала элемента системы, причем $A^{F}$ - множество автоматизированных задач, а $R^{F}$ - множество отношений между задачами; Р, О - множества ресурсов и организационнотехнических объектов системы гидроакустического мониторинга; $T=t_{K}-t_{H}-$ временной интервал.

Допустим, что возможности аппаратных компонентов и множество ресурсов системы гидроакустического мониторинга обеспечивают полную реализацию заданного функционала и соответствующее отображение обстановки. Цель функционирования системы достигается, если обеспечивается адекватное отображение подводной обстановки - взаимно-однозначное соответствие входного и выходного информационных объектов

$$
\mathrm{X} \Leftrightarrow \mathrm{X}_{\mathrm{R}} .
$$

Это соответствует условию

$$
\begin{aligned}
\left\{X \cap \overline{X_{R}}\right\} \cup\left\{\bar{X} \cap X_{R}\right\}= & 0, X_{R} \subseteq X, \\
& -210-
\end{aligned}
$$




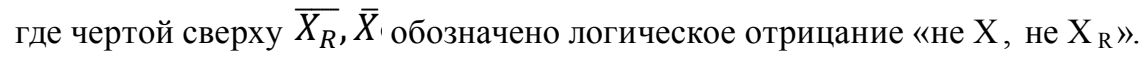

В реальных условиях система гидроакустического мониторинга должна обеспечить максимум количества элементов множества подводной обстановки, информация о которых в течение определенного времени получена (подверглась отображению и преобразованию оператором R) или минимизировать множество элементов, оставшихся без воздействия:

$$
\operatorname{card}\left\{\mathrm{X} \cap \overline{X_{R}}\right\} \cup\left\{\bar{X} \cap \mathrm{X}_{\mathrm{R}}\right\}=\operatorname{card} X_{l}, \rightarrow \min ,
$$

где $X_{l}$, $\operatorname{card} X_{l}$ - подмножество информационных потерь в системе $X_{l} \subseteq X$ и, соответственно, мощность этого подмножества.

К подмножеству информационных потерь отнесем потерянную в ходе сбора и обработки, ложную и дублирующую информацию.

Этим показателем, количественно характеризующим информационные потери при отображении, преобразовании входного информационного объекта в выходной, предлагается оценивать ее эффективность. Минимум информационных потерь обеспечивается при построении наиболее эффективной системы, поэтому критерием минимума информационных потерь можно оценивать степень достижения цели при агрегировании системы [6].

Таким образом, для повышения эффективности системы гидроакустического мониторинга с ограниченными ресурсами при ее формировании необходимо выбирать стратегию $A^{0 *}$, обеспечивающую достижение минимума информационных потерь:

$$
\begin{aligned}
& \mathrm{A}^{0 *}: \mathrm{G} \rightarrow K=\operatorname{minl}\left(S / F, R, P, A^{\circ}, Q^{*}\right), \\
& P \leq P_{\text {зад }},
\end{aligned}
$$

где $l$ - информационные потери; $F$ - множество показателей качества; $A^{0}$ - множество стратегий построения; $P, P_{\text {зад }}$ - множество показателей, характеризующих требуемые и заданные ресурсы системы; $Q^{*}$ - подмножество состояний среды, в которую погружена система.

По своей сути поставленная задача формирования системы гидроакустического мониторинга из компонентов представляет собой переход от функции многих переменных к функции или совокупности функций меньшего числа переменных, т.е. относится к классу задач агрегирования. Основные проблемы агрегирования связаны с определением условий, при которых можно избежать потерь информации.

Показатель информационных потерь обладает свойствами физического и функционального показателя. Он может рассчитываться с использованием результатов непосредственных измерений, полученных, например, в тестовом режиме, позволяет локализовать элементы системы, на которых происходят недопустимые потери. Этот показатель дает возмозность оценивать вклад информационной системы в надсистему и, отражая степень недостижения цели, может использоваться в качестве обратной связи при адаптации системы к состоянию среды. Кроме того, сформированный на его основе критерий минимума информационных потерь позволяет вполне целенаправленно проводить построение информационной системы в условиях неопределенности и обеспечивает сравнимость результатов оценки эффективности системы, полученных в ходе моделирования и применения по назначению [6].

$$
-211-
$$




\section{Список литературы / References}

[1] Оптнер С.А. Системный анализ для решения деловых и промышленных проблем. М.: Сов. радио, 1969. 215 с. [Optner S.A. System analysis to solve business and industrial problems, Moskva, Sov. Radio, 1969, 215 p. (in Russian)]

[2] Гафт М.Г. Принятие решений при многих критериях. М.: Знание, 1979. 520 c. [Gaft M.G. Multi-criteria decision making, Moskva, Znaniye, 1979, 520 p. (in Russian)]

[3] Андреев Г.И., Волчихин В.И., Миронов В.А., Тихомиров В.А. Основы управления предприятием. Т. 1. Современные взгляды на управление предприятием. М.: Финансы и статистика, 2004. 400 c. [Andreev G.I., Volchikhin V.I., Mironov V.A., Basics of enterprise management. Vol. 1. Modern views on enterprise management, Moskva, Finansy i statistika, 2004, 400 p. (in Russian)]

[4] Mesarovic M., Takahara Ya. General Systems Theory: Mathematical Foundations, Moskva, Mir, 1978, $311 \mathrm{p}$.

[5] Аверкин А.Н., Батыршин И.З., Блишун А.Ф., Силов В.Б., Тарасов В.Б. Нечеткие множества в моделях управления и искусственного интеллекта. М.: Наука, 1986. 312 c. [Averkin A.N., Batyrshin I.Z., Blishun A.F., Silov V.B., Tarasov V.B. Fuzzy sets in control and artificial intelligence models, Moskva, Nauka, 1986, 312 p. (in Russian)]

[6] Аксенов О.Ю., Кобан А.Я., Мельник Д.И., Семенов С.А, Семенов В.С. Агрегирование радиолокачионных систем и информации о воздушно-космической обстановке. Монография. M.: Знание, 2018. 224 c. [Aksyonov O.Yu., Koban A.Ya., Melnik D.I., Semenov S.A., Semenov V.S. Aggregation of radar systems and information on the aerospace situation. Monograph, Moskva, Znaniye, 2018, 224 p. (in Russian)] 